\title{
Frailty, Sarcopenia and Falls
}

\author{
Andréa Marques and Cármen Queirós
}

Research confirms that frailty, sarcopenia and falls are strongly correlated [1] and both are predictors of negative health outcomes such as falls, disability, hospitalisation and death [2]. Interventions are necessary to reverse frailty and treat sarcopenia [3] as it has been estimated that, by the year 2025, around $20 \%$ of the population in industrial countries will be aged 65 years and over. As the number of older people increases, their needs will become an increasingly important health issue. Reduction in physical function can lead to loss of independence, need for hospital and longterm nursing home care and premature death. The importance of physical, functional, psychological and social factors in realising a healthy old age is recognised by older people, health-care professionals, policy advisors and decision-makers.

This chapter will review the concepts of frailty, sarcopenia and falls as well as the interventions for older people, carried out by nurses and other health-care professionals, that have the potential to positively affect health and functional status and may promote independent functioning of older people with frailty and sarcopenia.

\subsection{Learning Outcomes}

At the end of the chapter, and following further study, the nurse will be able to:

\footnotetext{
A. Marques, R.N., M.Sc., Ph.D. ( $\bowtie)$

Rheumatology Department, Centro Hospitalar e Universitário de Coimbra, Coimbra, Portugal e-mail: amarques@reumahuc.org

C. Queirós

Nursing School of Coimbra, Esenf, Portugal

Escola Superior de Enfermagem do Porto, Porto, Portugal

e-mail: carmenqueiros@gmail.com
} 
- Identify individuals with frailty, low muscle mass and depleted strength

- Promote health and prevent ill health in older people with frailty and sarcopenia

- Plan interventions for patients with frailty and sarcopenia

- Educate older people about frailty, sarcopenia and fall prevention

- Promote correct nutrition and physical exercise in frail and sarcopenic patients.

\subsection{Frailty}

Frailty is a complex societal challenge of an ageing population and has significant repercussions for patient outcomes and health-care utilisation [4]. There is no universally accepted definition $[4,5]$, but experts agree that it is a clinical syndrome characterised by increased vulnerability and diminished resistance to stressors that can cause functional impairment and increase risks [6,7]; a minor stress or event such as an accidental fall or infection can worsen a person's health condition and increase dependency and/or mortality. Box 2.1 captures the main concepts in definitions of frailty.

Frailty can be physical or psychological or a combination of the two, with two common models used to explain it: (1) frailty is seen as a syndrome where sarcopenia (loss of muscle with ageing) is the main underlying concept [8] and individuals have at least three of a list of features including; unintentional weight loss, exhaustion, weakness, slowness and reduced physical activity and (2) frailty as the sum of an individual's deficits and non-specific disorders [9] that prevent individuals from launching an effective response to health stressors, leading to adverse health outcomes $[6,10]$.

Regardless of the perspective, frail patients are at increased risk of adverse health outcomes such as falls, hospitalisation, deterioration of mobility, disability, institutionalisation and death $[5,6,8]$, and assessing patients for frailty is an important aspect of the assessment process with several tools available for this. Epidemiological studies [11] have estimated the prevalence of frailty at between $4 \%$ and 59\%, depending on the population being studied [12], gender (higher in women than men) and age (the oldest have a higher prevalence) $[13,14]$.

\section{Box 2.1: Frailty Definition}

- Clinical syndrome

- Increased vulnerability

- Diminished resistance to stressors

- Can cause functional impairment

- Risk of adverse health outcomes 


\subsubsection{Assessment}

Early diagnosis of frailty can improve care and has an important role in preventing fractures in older adults [15]. All individuals over 70 years of age and all persons with unintentional and significant weight loss should be assessed for frailty [6]. Box 2.2. provides an overview of the most commonly used tools.

A comprehensive review identified 67 instruments for the assessment of frailty. Of these, nine were highly cited: the Physical Frailty Phenotype (PFP — also known as the Fried or CHS Frailty Phenotype), the Deficit Accumulation Index (DAI; also known as Frailty Index), the Gill Frailty Measure, the Frailty/Vigour Assessment, the Clinical Frailty Scale, the Brief Frailty Instrument, the Vulnerable Elders Survey (VES-13), the FRAIL Scale and the Winograd Screening Instrument. The selection of a specific instrument to assess frailty should be based on its purpose, theoretical approach, the validity of the constructs used and its feasibility in the clinical context [16]. More recently, an umbrella review was performed to identify the most valid, reliable and diagnostically accurate frailty screening tools [11], concluding that only a few frailty measures demonstrate these characteristics. Among them, the Frailty Index appeared as the most useful in standard care and community settings. However, the review could not identify an appropriate tool for assessing frailty in EDs, concluding that there is no universally appropriate screening tool for identifying frailty that could be recommended. It is important, however, to provide an overview of the most commonly used tools.

The Physical Frailty Phenotype (PFP, Fried or CHS Frailty Phenotype) was developed following observations of 5000 men and women aged $\geq 65$ years from the Cardiovascular Health Study [8]. This tool defines frailty as the presence of five criteria: weight loss ( $\geq 5 \%$ of body weight in the previous year), weakness (decreased grip strength), exhaustion (self-reported responses to questions about effort required for activity), slowness on walking (gait speed $\geq 6-7 \mathrm{~s}$ to walk 15 feet) and decreased physical activity (Kcal spent per week: males expending <383 Kcal and females $<270 \mathrm{Kcal}$ ) [8]. The assessment requires specialised equipment for grip strength measurement and involves patient participation to calculate gait speed. The PFP also facilitates identifying "pre-frailty"; one or two of the criteria for frailty are present.

The Deficit Accumulation or Frailty Index [9] is based on the individual's accumulated burden of illnesses, functional and cognitive decline and other health related deficits that, together, provide a flexible measurement of frailty. Deficits are measured by answering medical and functional related questions, allowing a frailty index to be quantified; the higher the number of deficits, the higher the frailty score. An assessment that identifies a score of 30-40 deficits has been shown to be able to predict adverse health outcomes [9, 17]. One advantage of using this tool, versus $\mathrm{PFP}$, is that it does not require a patient interview or exam, as the information can be retrieved from health records.

Some other instruments commonly used to assess frailty are quicker to use and, therefore, easier for nurses to apply; e.g. the Clinical Frailty Scale, FRAIL Scale 
and Study of Osteoporotic Fractures (SOF) frailty tool. The Clinical Frailty Scale uses pictographs and descriptors to categorise between very fit $(-1)$ and severely frail (-7). The assessment involves self-reporting (with no need for face-to-face examination) of comorbidities and the need for assistance with activities of daily living $[18,19]$. The scale is composed of five questions with "FRAIL" as an acronym: $\mathrm{F}=$ fatigue, $\mathrm{R}=$ resistance, $\mathrm{A}=$ ambulation, $\mathrm{I}=$ illnesses and $\mathrm{L}=$ loss of weight $[20,21]$; three or more positive answerers indicate frailty, and one or two positive answerers indicate pre-frailty. The Study of Osteoporotic Fractures (SOF) frailty tool assesses frailty according to three characteristics: loss of 5\% of body weight in the last year, inability to stand up from a chair five times without the use of arms and feeling full of energy; two positive answers to the first and second items and/or a negative to the last one classifies the person as frail [22].

\section{Box 2.2: Frailty Assessment}

- Individuals older than 70 years

- Individuals with unintentional and substantial weight loss $(\geq 5 \%)$

- The most common assessment tools are:

- Physical Frailty Phenotype

- Frailty Index

- Other instruments commonly used which are quicker and easier to adopt are:

- Clinical Frailty Scale

- FRAIL Scale

- Study of Osteoporotic Fractures (SOF) frailty tool

\subsubsection{Interventions}

Health-care interventions can help to improve the degree of frailty over time [6]. Evidence relates to four possible interventions (Box 2.3): (1) exercise (aerobic and resistance), (2) calorie and protein supplementation, (3) vitamin D supplementation and (4) reduction of polypharmacy $[6,14,21]$ :

- Planned exercise can develop muscle strength and improve physical performance and functionality [23] as well as decrease depression and fear of falling [6]. A mix of specifically prescribed aerobic and resistance exercises improves frailty and is effective in preventing its adverse outcomes [24, 25]. One systematic review found that an exercise programme, continued three times a week for 30-45 min per session for approximately 5 months, had positive impact [26].

- In frail older people with significant weight loss, it is essential to identify the cause (Chap. 8). Dietary calorific supplementation has been shown to be successful in achieving weight gain and reducing complications in malnourished individuals [27]. Protein supplementation of $15 \mathrm{~g}$ of protein twice a day over 24 weeks improves muscle strength and physical performance [28], while oral nutritional supplements provide additional protein and calories. 
- Vitamin D supplementation can play a role in preventing or treating frailty by enhancing balance and maintaining muscle strength [29] but, while this is likely to be beneficial for frail older people, there have been no large-scale studies that have confirmed this to be the case on its own [6].

- Undertaking a medication review and considering side effects, interactions and consequences for frailty is essential. Medication review and reduction of polypharmacy have also been advocated as an option for improving outcomes, especially in reducing mortality, hospital admissions and falls [30].

These four interventions should be considered following frailty assessment so that they can be individually tailored to target specific identified problems and needs [31].

\section{Box 2.3: Interventions}

- Exercise (aerobic and resistance)

- Caloric and protein supplementation

- Vitamin D supplementation

- Reduction of polypharmacy

\subsection{Sarcopenia}

Changes in body composition occur with normal physiological ageing [32]; usually, body weight increases during adulthood and peaks at the age of 65 years in women and 54 years in men [33]. Muscle mass is lost at a rate of approximately $8 \%$ per decade between the ages 50 and 70 years; then weight loss is coupled with an accelerated loss of muscle mass, reaching a rate of $15 \%$ each decade [33]. The overall prevalence of sarcopenia is reported to be $10 \%$ [34]; with the continued increase in the older population, sarcopenia is becoming a serious global public health problem.

Sarcopenia is associated with the ageing process [35]; loss of muscle mass and strength, which in turn affects balance, gait and overall ability to perform tasks of daily living, are hallmarks of this disease that is also a powerful predictor of disability [36]. The risk of disability is 1.5-4.6 times higher in older people with sarcopenia than in those with normal muscle. These common age-related changes in skeletal muscle are major causes of impaired physical function in older adults, contributing to impaired mobility, falls and hospitalisation. The causes of sarcopenia are multifactorial and can include muscle disuse, changing endocrine function, chronic diseases, inflammation, insulin resistance and nutritional deficiencies [38]; reductions in testosterone and oestrogen that accompany ageing appear to accelerate its development [39].

\subsubsection{Screening and Assessment for Sarcopenia}

Sarcopenia, like many other health conditions, is asymptomatic in its initial stages, when interventions can best prevent the adverse health outcomes [40]. Screening is 
currently not a routine aspect of clinical practice, partly because of the lack of appropriate screening strategies [41]. An ideal screening test should be cheap, acceptable and easily implementable without requiring additional training [42]. Several expert groups have convened with the goal of establishing a consensus about diagnostic criteria for sarcopenia [43-46]; a common theme is that diagnosis of sarcopenia should include identification of both low muscle mass and poor muscle function, indicated by either low muscle strength or impaired physical performance, such as slow gait speed. The European Working Group on Sarcopenia in Older People (EWGSOP) consensus outlined an algorithm to aid the screening and diagnosis of sarcopenia. Box 2.4 shows the diagnostic criteria. Patients with gait speeds of $0.8 \mathrm{~m} / \mathrm{s}$ or less should then undergo a second performance assessment, such as grip strength. Those meeting the criteria for low grip strength should be assessed by DXA (Chap. 1) or bioelectrical impedance analysis (BIA) to confirm the presence or absence of sarcopenia [44].

\section{Box 2.4: Diagnostic Criteria for Sarcopenia}

Sarcopenia should be considered in patients with presence of criteria 1 plus criteria 2 or 3 :

\section{Criteria 1: Low muscle mass}

DXA $>2$ SD below mean of the younger adults:

- Men $<7.26 \mathrm{~kg} / \mathrm{m}^{2}$

- Women $<5.5 \mathrm{~kg} / \mathrm{m}^{2}$

Lowest $20 \%$ of the distribution of appendicular skeletal mass (ASM) in a normative population (aged 65 years and older)

- Men $<7.23 \mathrm{~kg} / \mathrm{m}^{2}$

- Women $<5.67 \mathrm{~kg} / \mathrm{m}^{2}$

Lowest $20 \%$ distribution of the residual of ASM adjusting for height and fat mass

- Men $<2.29$

- Women: $<1.73$

BIA >2 SD below mean (SMI) of the younger adults

- Men $<8.87 \mathrm{~kg} / \mathrm{m}^{2}$

- Women $<6.42 \mathrm{~kg} / \mathrm{m}^{2}$

Criteria 2: Low grip strength

- Men: $<30 \mathrm{~kg}$

- Women: $<20 \mathrm{~kg}$

Criteria 3: Low physical performance

- Short Performance Battery (SPPB) $\leq 8$

- Gait speed $<0.8 \mathrm{~m} / \mathrm{s}$ 


\subsubsection{The Clinical Consequences of Sarcopenia}

Osteoporosis predicts the future risk of fracture; and sarcopenia is a powerful predictor of future disability [32]. Reduced muscle mass and strength are also associated with lower bone mineral density $[47,48]$, consistent with the "mechanostat" theory of bone loss due to reduced forces of muscle on bone [49]. In fact, sarcopenia may contribute to falls and, as a consequence, increase fracture risk [50, 51]. Hence, not surprisingly, there is evidence that low muscle mass and strength are associated with fractures [51]. Several studies have confirmed associations between low muscle mass, future functional decline and physical disability [2]. Physical inactivity or decreased physical activity is part of the underlying mechanisms of sarcopenia, so physical activity is important in reversing or modifying it. Several interventions have been proposed for the treatment of this loss of muscle and strength, but exercise is central. Sarcopenia has also been linked to higher hospitalisation rates, increased morbidity and mortality $[52,53]$. Sarcopenia may also be associated with metabolic and cardiovascular diseases such as diabetes, dyslipidaemia and hypertension [32].

\subsubsection{Interventions to Prevent Sarcopenia}

It is better to prevent progressive loss of skeletal muscle mass, strength and function rather than try to restore it later, so preventive strategies should be initiated early, before loss of skeletal muscle mass and strength occurs.

Exercise interventions have the most significant improvement in sarcopenia. The benefits of physical activity in the elderly population include lower mortality and functional independence (Chap. 6). There are four specific categories of recommended exercise: (1) aerobic exercise, (2) progressive resistance exercise, (3) flexibility exercise and (4) balance training [3].

Nutrition is also important in preventing and reversing sarcopenia (Chap. 7). Increasing age is associated with reduced appetite and early satiety, resulting in many older people failing to meet the recommended daily dietary allowance (RDA) for protein, which has important implications for skeletal muscles [54]. Older adults will require higher dietary protein (up to $1.2 \mathrm{~g} / \mathrm{kg} / \mathrm{day}$ ) to counteract age-related changes in protein metabolism and higher catabolic state associated with chronic or acute diseases [55].

It is the combination of exercise and nutrition interventions that is the key to preventing, treating and slowing down the progression of sarcopenia [54]. Pharmaceutical agents are under investigation but with no current proven benefit. Pharmacological agents such as myostatin inhibitors, testosterone, angiotensinconverting enzyme inhibitors and ghrelin-modulating agents are being investigated to treat sarcopenia, but there is inadequate evidence to support their use. Low serum vitamin D levels are associated with reduced muscle strength, and it has also been demonstrated that a dose-response relationship exists between serum levels and muscle health. If serum levels are low, vitamin D should be replaced with replenishment dosages ranging from 700 to 1000 IU/day [56]. 


\section{Box 2.5: Multiple Factors That Contribute Collectively to Frailty, Sarcopenia and Falls \\ Potentially treatable:}

- Social factors including social isolation, living alone

- Lack of access to transport

- Elder abuse

- Poverty and food insecurity

- Failure to provide for ethnic food preference

- Inability to prepare and cook meals or to feed self

- Inability to shop

- Alcoholism

\section{Medical:}

- Thyroid disease

- Cardiac failure

- Gastrointestinal disease affecting absorption: anorexia (antibiotics/ digoxin), early satiety (anticholinergic drugs), reduced feeding ability (such as sedatives/psychotropics), dysphagia (NSAIDs), constipation (opiates/diuretics), diarrhoea (laxatives/antibiotics), hypermetabolism (thyroxin)

- Sensory impairment-vision/hearing

- Oral problem, e.g. poorly fitting dentures

- Swallowing problem/dysphagia, thickened diet

- Poorly managed pain or constipation

More difficult to treat:

- Medical factors

- Loss of taste and smell, restricted diets

- Cognition-dementia

- Catabolism

- Gastritis

- Cancer

- Mood-depression, paranoia

- Medications/polypharmacy

Implementing interventions for frailty and sarcopenia has several challenges and barriers. A systematic review demonstrated that older people believe that exercise is unnecessary or, even, potentially harmful [58]. Others recognise the benefits of exercise but report a range of barriers to participation in exercise interventions. Raising awareness is important to enhance exercise participation among older people and to prevent sarcopenia. 
Another barrier that needs to be considered in planning long-term strategies to prevent and treat sarcopenia in older people is the financial ability to attend exercise programmes [59]. Factors such as access to food, finances and social isolation may all impact on an older person's ability to obtain optimal food intake.

\subsection{The Link Between Frailty, Sarcopenia and Falls}

Falls in older people are associated with multicomponent impairments, particularly of muscle function, balance and cognition, so are best understood as resulting from complex system failure as part of the frailty syndrome in the presence of sarcopenia [57]. Falls and fall prevention are considered in more detail in Chap. 3. Box 2.5 provides an overview of the multiple factors that contribute collectively to frailty, sarcopenia and falls, which include cellular and tissue changes, as well as environmental and behavioural factors.

\subsection{Suggested Further Study}

Search for information and online programmes on the impact of ageing on older people:

- http://aginginmotion.org/

- https://nos.org.uk/for-health-professionals/

- https://www.cme.nof.org/

Talk with patients, carers and other staff about the things they feel that lead to and prevent frailty, sarcopenia and falls. Reflect on what these conversations suggest about how practice might be developed to improve mobility outcomes by involving patients.

\subsection{How to Self-Assess Learning}

- Seek advice and mentorship from other expert clinicians.

- Meet with specialists and other members of the team to keep up to date on new evidence and disseminate it to colleagues.

- Search on a regular basis about recent new practices, guidance, knowledge or evidence.

\section{References}

1. Reijnierse EM et al (2016) Common ground? the concordance of sarcopenia and frailty definitions. J Am Med Dir Assoc 17(4):371.e7-371.12

2. Beaudart Cet al (2017) Health outcomes of sarcopenia: a systematic review and meta-analysis. PLoS One 12(1):e0169548 
3. Lozano-Montoya I et al (2017) Nonpharmacological interventions to treat physical frailty and sarcopenia in older patients: a systematic overview-the SENATOR Project ONTOP Series. Clin Interv Aging 12:721-740

4. Sutton JL et al (2016) Psychometric properties of multicomponent tools designed to assess frailty in older adults: a systematic review. BMC Geriatr 16:55

5. Lohman MC et al (2017) Depression and frailty: concurrent risks for adverse health outcomes. Aging Ment Health 21(4):399-408

6. Morley JE et al (2013) Frailty consensus: a call to action. J Am Med Dir Assoc 14(6):392-397

7. Rodriguez-Manas L et al (2013) Searching for an operational definition of frailty: a Delphi method based consensus statement: the frailty operative definition-consensus conference project. J Gerontol A Biol Sci Med Sci 68(1):62-67

8. Fried LP et al (2001) Frailty in older adults: evidence for a phenotype. J Gerontol A Biol Sci Med Sci 56(3):M146-MM57

9. Rockwood K, Mitnitski A (2007) Frailty in relation to the accumulation of deficits. J Gerontol A Biol Sci Med Sci 62(7):722-727

10. Chowdhury R et al (2017) Frailty and chronic kidney disease: a systematic review. Arch Gerontol Geriatr 68:135-142

11. Apóstolo J et al (2017) Predicting risk and outcomes for frail older adults: an umbrella review of frailty screening tools. JBI Database System Rev Implement Rep 15(4):1154-1208

12. Wilson MG et al (2015) Interventions for preventing, delaying the onset, or decreasing the burden of frailty: an overview of systematic reviews. Syst Rev 4:128

13. Collard RM et al (2012) Prevalence of frailty in community-dwelling older persons: a systematic review. J Am Geriatr Soc 60(8):1487-1492

14. Tello-Rodriguez T, Varela-Pinedo L (2016) Frailty in older adults: detection, communitybased intervention, and decision-making in the management of chronic illnesses. Rev Peru Med Exp Salud Publica 33(2):328-334

15. Wei Chen K, Chang S-F (2017) Frailty was related with fracture: a systematic review. Int J Nurs Health Sci 3(1):1-4

16. Buta BJ et al (2016) Frailty assessment instruments: systematic characterization of the uses and contexts of highly-cited instruments. Ageing Res Rev 26:53-61

17. Searle SD et al (2008) A standard procedure for creating a frailty index. BMC Geriatr 8:24

18. Juma S (2016) Clinical Frailty Scale in an Acute Medicine Unit: a Simple Tool That Predicts Length of Stay. Can Geriatr J 19(2):34-39

19. Rockwood K et al (2005) A global clinical measure of fitness and frailty in elderly people. CMAJ 173(5):489-489

20. Morley JE et al (2012) A simple frailty questionnaire (FRAIL) predicts outcomes in middle aged African Americans. J Nutr Health Aging 16(7):601-608

21. Morley JE (2014) Frailty screening comes of age. J Nutr Health Aging 18(5):453-454

22. Silva $J$ et al (2017) Impact of insomnia on self-perceived health in the elderly. Arq Neuropsiquiatr 75(5):277-281

23. Cruz-Jentoft AJ (2014) Prevalence of and interventions for sarcopenia in ageing adults: a systematic review. Report of the International Sarcopenia Initiative (EWGSOP and IWGS). Age Ageing 43(6):748-759

24. Marzetti E et al (2017) Physical activity and exercise as countermeasures to physical frailty and sarcopenia. Aging Clin Exp Res 29(1):35-42

25. Cesari $\mathrm{M}$ et al (2015) A physical activity intervention to treat the frailty syndrome in older persons-results from the LIFE-P study. J Gerontol A Biol Sci Med Sci 70(2):216-222

26. Theou $\mathrm{O}$ et al (2011) The effectiveness of exercise interventions for the management of frailty: a systematic review. J Aging Res 569194

27. Milne AC et al (2009) Protein and energy supplementation in elderly people at risk from malnutrition. Cochrane Database Syst Rev. 2009;(2):CD003288. doi: https://doi. org/10.1002/14651858.CD003288.pub3.

28. Tieland $\mathrm{M}$ et al (2012) Protein supplementation improves physical performance in frail elderly people: a randomized, double-blind, placebo-controlled trial. J Am Med Dir Assoc 13(8):720-726 
29. Montero-Odasso M, Duque G (2005) Vitamin D in the aging musculoskeletal system: an authentic strength preserving hormone. Mol Aspects Med 26(3):203-219

30. Romera L et al (2014) Effectiveness of a primary care based multifactorial intervention to improve frailty parameters in the elderly: a randomised clinical trial: rationale and study design. BMC Geriatr 14:125

31. Cameron ID et al (2013) A multifactorial interdisciplinary intervention reduces frailty in older people: randomized trial. BMC Med 11:65

32. Shaw SC (2017) Epidemiology of sarcopenia: determinants throughout the lifecourse. Calcif Tissue Int 101(3):229-247

33. Yu S et al (2014) Sarcopenia in older people. Int J Evid Based Healthc 12(4):227-243

34. Shafiee $G$ et al (2017) Prevalence of sarcopenia in the world: a systematic review and metaanalysis of general population studies. J Diabetes Metab Disord 16:21

35. Steen B (1988) Body Composition and Aging. Nutr Rev 46(2):45-51

36. Morley JE (2016) Frailty and Sarcopenia: the new geriatric giants. Rev Invest Clin 68(2):59-67

37. Janssen I et al (2004) The healthcare costs of sarcopenia in the United States. J Am Geriatr Soc 52(1):80-85

38. Janssen I (2011) The epidemiology of sarcopenia. Clin Geriatr Med 27(3):355-363

39. Joseph $\mathrm{C}$ et al (2005) Role of endocrine-immune dysregulation in osteoporosis, sarcopenia, frailty and fracture risk. Mol Aspects Med 26(3):181-201

40. McLean RR, Kiel DP (2015) Developing consensus criteria for sarcopenia: an update. J Bone Miner Res 30(4):588-592

41. Studenski SA et al (2014) The FNIH sarcopenia project: rationale, study description, conference recommendations, and final estimates. J Gerontol A Biol Sci Med Sci 69(5):547-558

42. Fields MM, Chevlen E (2006) Screening for disease: making evidence-based choices. Clin J Oncol Nurs 10(1):73-76

43. Muscaritoli M et al (2010) Consensus definition of sarcopenia, cachexia and pre-cachexia: joint document elaborated by Special Interest Groups (SIG). Cachexia-anorexia in chronic wasting diseases" and "nutrition in geriatrics". Clin Nutr 29(2):154-159

44. Cruz-Jentoft AJ et al (2010) Sarcopenia: European consensus on definition and diagnosis: report of the European Working Group on Sarcopenia in Older People. Age Ageing 39(4):412-423

45. Fielding RA et al (2011) Sarcopenia: an undiagnosed condition in older adults. Current consensus definition: prevalence, etiology, and consequences. International working group on sarcopenia. J Am Med Dir Assoc 12(4):249-256

46. Morley JE et al (2011) Sarcopenia with limited mobility: an international consensus. J Am Med Dir Assoc 12(6):403-409

47. Proctor DN et al (2000) Relative influence of physical activity, muscle mass and strength on bone density. Osteoporos Int 11(11):944-952

48. Singh $\mathrm{H}$ et al (2017) Relationship between muscle performance and DXA-derived bone parameters in community-dwelling older adults. J Musculoskelet Neuronal Interact 17(2):50-58

49. Frost HM (2003) Bone's mechanostat: a 2003 update. Anat Rec A Discov Mol Cell Evol Biol 275(2):1081-1101

50. Landi F et al (2012) Sarcopenia as a risk factor for falls in elderly individuals: results from the ilSIRENTE study. Clin Nutr 31(5):652-658

51. Cederholm T et al (2013) Sarcopenia and fragility fractures. Eur J Phys Rehabil Med 49(1):111-117

52. Oakland $\mathrm{K}$ et al (2016) Systematic review and meta-analysis of the association between frailty and outcome in surgical patients. Ann R Coll Surg Engl 98(2):80-85

53. Wang SY et al (2013) Not just specific diseases: systematic review of the association of geriatric syndromes with hospitalization or nursing home admission. Arch Gerontol Geriatr 57(1):16-26

54. Deutz NE et al (2014) Protein intake and exercise for optimal muscle function with aging: recommendations from the ESPEN Expert Group. Clin Nutr 33(6):929-936

55. Bauer J et al (2013) Evidence-based recommendations for optimal dietary protein intake in older people: a position paper from the PROT-AGE Study Group. J Am Med Dir Assoc 14(8):542-559 
56. Bischoff Ferrari HA (2009) Validated treatments and therapeutic perspectives regarding nutritherapy. J Nutr Health Aging 13(8):737-741

57. Boirie Y (2009) Physiopathological mechanism of sarcopenia. J Nutr Health Aging 13(8):717-723

58. Franco MR et al (2015) Older people's perspectives on participation in physical activity: a systematic review and thematic synthesis of qualitative literature. Br J Sports Med 49(19):1268-1276

59. Freiberger E (2011) Physical activity, exercise, and sarcopenia—future challenges. Wien Med Wochenschr 161(17-18):416-425

Open Access This chapter is licensed under the terms of the Creative Commons Attribution 4.0 International License (http://creativecommons.org/licenses/by/4.0/), which permits use, sharing, adaptation, distribution and reproduction in any medium or format, as long as you give appropriate credit to the original author(s) and the source, provide a link to the Creative Commons license and indicate if changes were made.

The images or other third party material in this chapter are included in the chapter's Creative Commons license, unless indicated otherwise in a credit line to the material. If material is not included in the chapter's Creative Commons license and your intended use is not permitted by statutory regulation or exceeds the permitted use, you will need to obtain permission directly from the copyright holder.

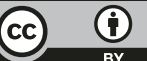

